\title{
Nanophotonic Optical Isolator Controlled by the Internal State of Cold Atoms
}

\author{
Clément Sayrin, Christian Junge, Rudolf Mitsch, Bernhard Albrecht, Danny O’Shea, \\ Philipp Schneeweiss, ${ }^{\dagger}$ Jürgen Volz, ${ }^{\ddagger}$ and Arno Rauschenbeutel ${ }^{\S}$ \\ Vienna Center for Quantum Science and Technology, Atominstitut, TU Wien, \\ Stadionallee 2, 1020 Vienna, Austria
}

(Received 12 June 2015; revised manuscript received 16 September 2015; published 4 December 2015)

\begin{abstract}
The realization of nanophotonic optical isolators with high optical isolation even at ultralow light levels and low optical losses is an open problem. Here, we employ the link between the local polarization of strongly confined light and its direction of propagation to realize low-loss nonreciprocal transmission through a silica nanofiber at the single-photon level. The direction of the resulting optical isolator is controlled by the spin state of cold atoms. We perform our experiment in two qualitatively different regimes, i.e., with an ensemble of cold atoms where each atom is weakly coupled to the waveguide and with a single atom strongly coupled to the waveguide mode. In both cases, we observe simultaneously high isolation and high forward transmission. The isolator concept constitutes a nanoscale quantum optical analog of microwave ferrite resonance isolators, can be implemented with all kinds of optical waveguides and emitters, and might enable novel integrated optical devices for fiber-based classical and quantum networks.
\end{abstract}

DOI: 10.1103/PhysRevX.5.041036

\section{INTRODUCTION}

Miniaturized components that control the flow of light are key to information processing in integrated optical circuits. When it comes to power consumption and channel capacity, such circuits have the potential to largely outperform their electronic counterparts [1]. In these structures, the wiring is realized with nanophotonic waveguides. Thus, the control of the direction of the light flow requires nanophotonic nonreciprocal devices, i.e., components that exhibit an inherent asymmetry between light propagation in the forward and the backward directions. Among those, components that yield an asymmetric transmission, like optical diodes and circulators, are of particular interest. Beyond their relevance for classical optical circuits, such devices are essential also for future quantum networks [2,3] where information is encoded in single photons. In order to be suitable for such quantum applications, however, these

\footnotetext{
*Present address: Zernike Institute for Advanced Materials, University of Groningen, Netherlands.

Corresponding author. schneeweiss@ati.ac.at

${ }^{\ddagger}$ Corresponding author. jvolz@ati.ac.at

${ }^{\S}$ Corresponding author. arno.rauschenbeutel@ati.ac.at
}

Published by the American Physical Society under the terms of the Creative Commons Attribution 3.0 License. Further distribution of this work must maintain attribution to the author(s) and the published article's title, journal citation, and DOI.
Subject Areas: Atomic and Molecular Physics, Photonics, Quantum Physics

elements must exhibit low optical losses and be compatible with ultralow light levels.

Commercially available Faraday isolators fulfill the above requirements but are bulk optical components. Their miniaturization is hampered by the birefringence of typical integrated waveguides [4]. Integrated isolators that make use of nonlinear optical effects in waveguides [5] or microcavities [6,7] have been demonstrated. However, they require large intensities and thus cannot operate at the single-photon level. Using time modulation of the waveguide properties, nonreciprocal transmission at low light levels has been achieved [8-11], yet only demonstrating an isolation of less than $3 \mathrm{~dB}$.

In the microwave domain, very effective integrated isolators and circulators exist and typically employ the inherent link between the local polarization and the propagation direction of the guided fields [12]. This effect is most pronounced for a field confinement at the subwavelength scale. It locally gives the photons a chiral character and is sometimes referred to as spin-orbit interaction [13]. Coupling these chiral fields to magneto-optical materials that exhibit polarization-dependent permeabilities when exposed to an external magnetic field [14] then yields nonreciprocal behavior [15]. Significant research is invested into adapting such schemes for integrated optics [16-19]. While providing high optical isolation, state-ofthe-art implementations, however, still suffer from high insertion losses. The realization of miniaturized, integrated optical isolators that simultaneously provide high optical isolation even at ultralow light levels and low optical losses is still an open problem. 
Recently, the interaction of quantum emitters with light fields that exhibit spin-orbit interaction has been observed in the strongly confined optical modes of whisperinggallery-mode (WGM) resonators $[20,21]$ and nanoscale waveguides [22-24]. This opens the route towards a new class of nonreciprocal devices in which the quantum state of the emitter controls the light propagation in nanophotonic waveguides [25-27]. Moreover, by optically addressing the quantum emitters, these devices could be programmed and actively reconfigured. Nowadays, quantum emitters, like single atoms, molecules, quantum dots, or color centers, can be prepared and manipulated with high precision. This should, therefore, allow bottom-up engineering of nonreciprocal components, like diodes and circulators, from their microscopic constituents.

Here, we demonstrate low-loss silica nanophotonic waveguides with a strongly nonreciprocal transmission controlled by the internal state of spin-polarized atoms. In a first experiment, an ensemble of atoms interacts with light guided in an optical nanofiber [28]. Here, each atom is weakly coupled to the waveguide. In a second experiment, the diode is controlled by a single atom that is strongly coupled to the waveguide by means of an ultrahigh-quality factor WGM bottle microresonator [20]. With the atomic ensemble, we measure an imbalance between the transmissions in the forward and the backward direction as large as $8 \mathrm{~dB}$ for a few ten atoms, while it is $13 \mathrm{~dB}$ with the resonator-enhanced scheme. At the same time, the forward transmissions remain as high as $78 \%$ and $72 \%$, respectively. Both experiments are carried out in an effective single-photon regime, i.e., a regime where every quantum emitter interacts with at most one photon at a time.

\section{CHIRAL INTERACTION BETWEEN ATOMS AND LIGHT}

\section{A. Chiral photons in optical nanofibers}

Light that is transversally confined at the subwavelength scale can exhibit a significant polarization component along the propagation direction. The latter oscillates in phase quadrature with respect to the transverse components. Thus, confined quasilinearly polarized light exhibits a local spin that is transverse, i.e., orthogonal to the propagation direction of the field [20,29]. This occurs, e.g., in the evanescent field that surrounds an optical nanofiber [23]; see Fig. 1(a). When the evanescent field propagates in the $(+z)$ direction, it is almost fully $\sigma^{+}$polarized if the $y$ axis is taken as the quantization axis. However, it is almost fully $\sigma^{-}$polarized if it propagates in the $(-z)$ direction. This shows that photons in an evanescent field have chiral character: there is an inherent link between their local polarization and their propagation direction.

In order to quantify this chiral character, we locally define an effective chirality $\chi=\boldsymbol{\varepsilon} \cdot\left(\boldsymbol{k} /|\boldsymbol{k}| \times \mathbf{e}_{r}\right)$, where $\mathbf{e}_{r}$ is the normal vector of the surface and $\boldsymbol{k}$ is the wave vector,

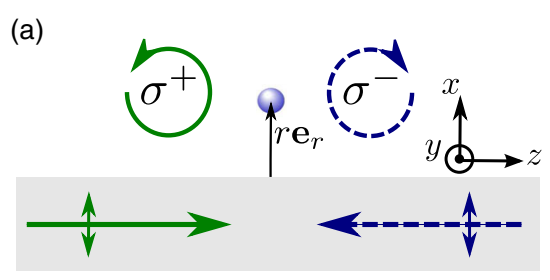

(b)

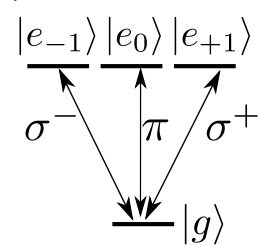

FIG. 1. Chiral photons in evanescent fields coupled to spinpolarized atoms. (a) Polarization properties of the evanescent light field that surrounds an optical nanofiber (gray). A light field that propagates in the $(+z)$ direction and whose main polarization axis (double arrow) is along the $x$ axis is almost fully $\sigma^{+}$polarized (green solid arrows) in the $(y=0)$ plane. If it propagates in the $(-z)$ direction, it is almost fully $\sigma^{-}$polarized (blue dashed arrows). The quantization axis is chosen along $y$, i.e., orthogonal to the propagation direction. An atom (light blue sphere) placed at a distance $r$ to the nanofiber surface couples to the evanescent field. (b) Relevant energy levels of the atom. The ground state $|g\rangle$ is coupled to the excited states $\left|e_{-1}\right\rangle,\left|e_{0}\right\rangle$, and $\left|e_{+1}\right\rangle$ via $\sigma^{-}, \pi$, and $\sigma^{+}$transitions, respectively.

$\varepsilon=i\left(\mathcal{E}^{*} \times \mathcal{E}\right) /|\mathcal{E}|^{2}$ is the local ellipticity vector, or spin, and $\mathcal{E}$ is the positive-frequency envelope of the electric field. In contrast to plane waves where this chirality is always zero, photons in evanescent fields have positive chirality, for both the $(+z)$ - and $(-z)$-propagation directions.

\section{B. From chiral photons to nonreciprocal waveguides}

The chiral character of photons has been demonstrated in recent experiments by means of a polarization-dependent directional scattering by gold nanoparticles [30,31], nanotips [32], a silicon microdisk [33], quantum dots [22,24], and atoms [23]. This chiral character and the resulting directional scattering are, however, not sufficient to realize nonreciprocal optical elements. For this purpose, Lorentz reciprocity [34] has to be broken. For example, in Faraday isolators, nonreciprocity stems from the change of the sign of a magnetic field $\mathbf{B}$ under time reversal.

In our experiment, we demonstrate that the chiral nature of photons can be exploited for the realization of an optical diode when the photons interact with spin-polarized atoms. Such atoms act as polarization-dependent scatterers, i.e., exhibit different interaction cross sections for $\sigma^{+}$- and $\sigma^{-}$-polarized light. The resulting chiral interaction between the atoms and the guided light leads to nonreciprocal transmission as conceptually discussed in Refs. [25-27]. We note that the interaction cross sections for $\sigma^{+}$- and $\sigma^{-}$-polarized light, and thus their ratio, depend on the angular momentum quantum numbers of the atoms but not on the respective Landé $g$ factors [35]. The strength of the nonreciprocity is therefore independent of the magnitude of the involved magnetic moments and their associated magnetic fields. Our concept thus fundamentally differs from other isolator schemes because it relies on the atomic spin to break Lorentz reciprocity. 
The relevant energy levels of the atoms are given in Fig. 1(b). The light field is assumed to be close to resonance with the transitions from the ground state $|g\rangle$ to the excited states $\left|e_{i}\right\rangle$, where $i \in\{-1,0,+1\}$ denotes the change in magnetic quantum number of the atom with respect to $|g\rangle$. Considering the simplified case where the light is perfectly circularly polarized at the position of the atoms, i.e., $\chi=|\varepsilon|=1$, the $|g\rangle \rightarrow\left|e_{+1}\right\rangle$ and $|g\rangle \rightarrow\left|e_{-1}\right\rangle$ transitions are driven only when the light field propagates in the $(+z)$ and $(-z)$ directions, respectively. The $|g\rangle \rightarrow\left|e_{0}\right\rangle$ transition is not driven. If we choose the atomic spin state $|g\rangle$ such that the two transitions $|g\rangle \rightarrow\left|e_{ \pm 1}\right\rangle$ have significantly different strengths, the atoms couple unequally to $\sigma^{+}$and $\sigma^{-}$-polarized light. As a consequence, one obtains a strong dependence of the atom-light coupling strength on the propagation direction of the chiral photons. In order to characterize the coupling between the atoms and the light fields that propagate in the $(+z)$ and $(-z)$ direction, we introduce the coefficients $\beta_{+}$and $\beta_{-}$, respectively. They are defined as $\beta_{ \pm}=\sum_{i} \beta_{ \pm}^{(i)}$, where

$$
\beta_{ \pm}^{(i)}=\frac{\kappa_{ \pm}^{(i)}}{\kappa_{+}^{(i)}+\kappa_{-}^{(i)}+\gamma^{(i)}}, \quad i \in\{-1,0,+1\}
$$

Here, $\kappa_{ \pm}^{(i)}$ is the spontaneous emission rate of an atom in the state $\left|e_{i}\right\rangle$ into the light mode that propagates in the $( \pm z)$ direction and $\gamma^{(i)}$ accounts for all other loss channels such as spontaneous emission into free space. In the ideal case considered above, $\kappa_{ \pm}^{(0)}=\kappa_{+}^{(-1)}=\kappa_{-}^{(+1)}=0$ and $\kappa_{+}^{(+1)} \neq \kappa_{-}^{(-1)}$. Therefore, $\beta_{+}=\beta_{+}^{(+1)}$ differs from $\beta_{-}=\beta_{-}^{(-1)}$, i.e., the atom-light interaction is nonreciprocal.

\section{EXPERIMENTAL DEMONSTRATION}

\section{A. Nanofiber-trapped ensemble of cold atoms}

We first use an ensemble of individual cesium atoms located in the vicinity of a subwavelength-diameter silica nanofiber; see Fig. 2. The atoms are trapped in a nanofiberbased two-color optical dipole trap that consists of two diametric linear arrays of trapping sites [28]. These sites are located $230 \mathrm{~nm}$ from the nanofiber surface and contain at most one atom [36]. Here, for technical reasons, we use only the atoms in one of the two linear arrays (see Appendix B), namely, in the one located at $x>a$, where $a=250 \mathrm{~nm}$ is the radius of the nanofiber; see Fig. 2(b).

We send a quasilinearly polarized light field [37] that is resonant with the $F=4 \rightarrow F^{\prime}=5(\lambda=852 \mathrm{~nm})$ transition of the cesium $D 2$ line through the nanofiber, where $F$ is the value of the atomic spin. When its main polarization is along the $x$ axis, see Fig. 2(b), the guided photons exhibit the required chirality: At the position of the atoms, the local chirality takes its maximum value of $\chi_{\max }=0.84$. In the case where the main polarization axis is along the $y$ axis,

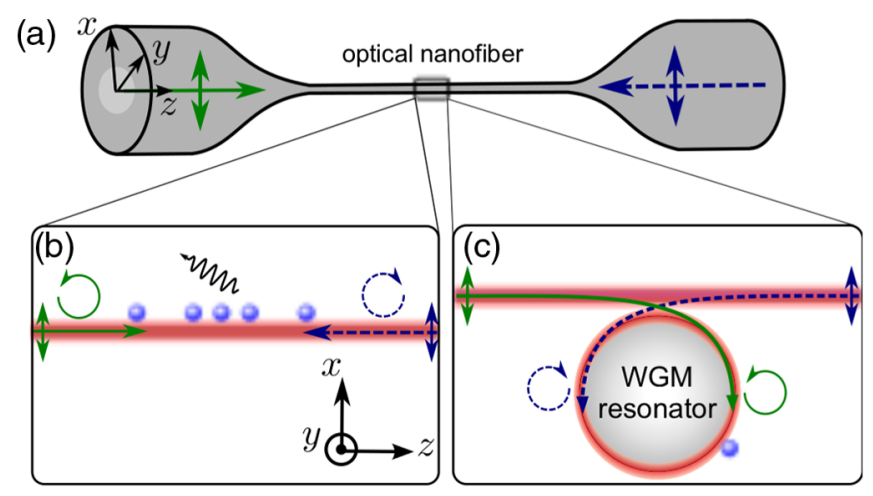

FIG. 2. Schematics of the demonstrated nonreciprocal waveguides. (a) An optical nanofiber is realized as the waist of a tapered silica fiber. (b) Atoms are trapped in the vicinity of the nanofiber, interact with the evanescent field of the nanofiberguided modes, and scatter light out of the nanofiber (wavy arrow) with a rate that depends on the direction of propagation. (c) A single atom is coupled to a whispering-gallery-mode (WGM) bottle microresonator. The atom-resonator coupling strength depends on the propagation direction of the field in the WGM resonator. The WGM resonator is coupled to the optical nanofiber via frustrated total internal reflection.

the evanescent field is purely linearly polarized at the position of the atoms [23] and $\chi=0$.

Using nanofiber-guided light, the atoms are initially optically pumped towards the state $|g\rangle=\left|F=4, m_{F}=+4\right\rangle$ [38], where $m_{F}$ is the projection of the atomic spin $F$ on the quantization axis. In order to suppress spin flips due to the polarization gradients of the trapping light fields, and, thus, to maintain the Zeeman state, a magnetic offset field $B$ is applied along the $y$ axis [39]. For probe light with its main polarization along the $x$ axis that propagates in the $( \pm z)$ direction, a fraction $\left(1 \pm \chi_{\max }\right) / 2$ of the light couples to the $\left|e_{+1}\right\rangle$ state, while a fraction $\left(1 \mp \chi_{\max }\right) / 2$ couples to the $\left|e_{-1}\right\rangle$ state. Here, $\left|e_{+1}\right\rangle=\left|F^{\prime}=5, m_{F}=+5\right\rangle,\left|e_{0}\right\rangle=$ $\left|F^{\prime}=5, m_{F}=+4\right\rangle$, and $\left|e_{-1}\right\rangle=\left|F^{\prime}=5, m_{F}=+3\right\rangle$. With this choice, the $|g\rangle \rightarrow\left|e_{+1}\right\rangle$ transition is 45 times stronger than the $|g\rangle \rightarrow\left|e_{-1}\right\rangle$ transition. Furthermore, in this experiment, $B=28 \mathrm{G}$, and the laser light is made resonant with the $|g\rangle \rightarrow\left|e_{+1}\right\rangle$ transition. The $|g\rangle \rightarrow\left|e_{-1}\right\rangle$ transition is then detuned by $31 \mathrm{MHz}-\mathrm{a}$ detuning much bigger than the natural linewidth of the $\left|e_{ \pm 1}\right\rangle$ states of $2 \gamma^{( \pm 1)}=2 \pi \times 5.2 \mathrm{MHz}$. This detuning and the difference of transition strengths then play a similarly important role in rendering the coupling to the state $\left|e_{-1}\right\rangle$ negligible compared to the coupling to the state $\left|e_{+1}\right\rangle$. For our value of $\chi_{\max }=0.84$, this even prevails when the photons propagate in the $(-z)$ direction. The guided light field is thus coupled to an effective two-level atom and $\beta_{+}^{(-1)}=\beta_{-}^{(-1)}=0$, $\beta_{+}=\beta_{+}^{(+1)}$, and $\beta_{-}=\beta_{-}^{(+1)}$. In this situation, the power transmission of a weak coherent light field that propagates in the $( \pm z)$ direction, for a single coupled atom, is given by $\mathcal{T}_{ \pm}=\left|t_{ \pm}\right|^{2}$, where $t_{ \pm}=1-2 \beta_{ \pm}^{(+1)}$ [27]. Remarkably, 
$\beta_{+}^{(+1)} / \beta_{-}^{(+1)}=\left(1+\chi_{\max }\right) /\left(1-\chi_{\max }\right)=11.5$ and strongly nonreciprocal transmission through the nanofiber is made possible.

In our experiment, $\beta_{+}^{(+1)} \approx 0.025 \ll 1$ [40]. This means that each atom absorbs a few percent of the nanofiberguided probe light field and that almost all photons that are emitted by the atoms are scattered into free space. With a single trapped atom, one therefore expects an isolation $\mathcal{I}=10\left|\log \left(\mathcal{T}_{-} / \mathcal{T}_{+}\right)\right| \approx 0.3 \mathrm{~dB}$. In order to increase the isolation, our scheme requires several atoms to interact with the nanofiber-guided light: With $N$ atoms, the transmissions read $\mathcal{T}_{ \pm}=\left(\left|t_{ \pm}\right|^{2}\right)^{N}$. The isolation thus scales linearly with $N$.

In order to measure the transmissions $\mathcal{T}_{ \pm}$, two singlephoton-counting modules are placed at the two ends of the nanofiber. The number of transmitted photons is measured during $300 \mu \mathrm{s}$, with and without trapped atoms, and is corrected for background counts. The transmissions $\mathcal{T}_{ \pm}$are given by the ratio of these two numbers. With a power of the probe light field of $0.8 \mathrm{pW}$, corresponding to $\approx 0.1$ photon per excited-state lifetime, we find the transmissions $\mathcal{T}_{+}=0.13 \pm 0.01$ and $\mathcal{T}_{-}=0.78 \pm 0.02$. This strongly nonreciprocal transmission corresponds to an isolation $\mathcal{I}=7.8 \mathrm{~dB}$ - the residual absorption in the $(-z)$ direction is due to the fact that $\chi<1$. Our scheme thus enables simultaneously high transmission in the $(-z)$ direction and high isolation.

The local chirality $\chi$ of the photons that interact with the atoms can be continuously tuned via the orientation of the main polarization axis. The latter is labeled by the angle $\varphi$; see inset of Fig. 3. When $\varphi=0, \chi$ reaches its maximum of 0.84 , while it vanishes when $\varphi=90^{\circ}$. In Fig. 3, we plot the values of $\mathcal{T}_{+}, \mathcal{T}_{-}$and $\mathcal{I}$ for different values of $\chi$. When $\chi$ is decreased, the isolation decreases. Ultimately, it reaches $\mathcal{I}=0 \mathrm{~dB}$ for $\chi=0$, i.e., the transmission becomes symmetric. The evanescent field then couples to the $|g\rangle \rightarrow\left|e_{0}\right\rangle$ transition for both propagation directions. Here, because of the applied magnetic field, the light field is out of resonance with the $|g\rangle \rightarrow\left|e_{0}\right\rangle$ transition and $\mathcal{T}_{+}=\mathcal{T}_{-} \approx 1$. We can thus control whether the transmission through the nanofiber should be nonreciprocal or reciprocal by choosing $\varphi=0^{\circ}$ or $90^{\circ}$, respectively. If required, the propagation of light polarized along $\varphi=90^{\circ}$ can simply be suppressed by adding a polarization filter.

Our measurements are in very good agreement with theoretical predictions calculated for our experimental parameters and a mean number of trapped atoms $\langle N\rangle \approx 27$; see solid lines in Fig. 3 and Table I. Here, $N$ is assumed to follow a Poisson distribution. We note that even without a magnetic offset field, i.e., when the two $|g\rangle \rightarrow\left|e_{ \pm 1}\right\rangle$ transitions are degenerate, one expects a similar performance of the diode; see theoretical predictions in Table I. In this case, the nonreciprocal transmission relies solely on the significant difference between the strengths of the $|g\rangle \rightarrow\left|e_{+1}\right\rangle$ and $|g\rangle \rightarrow\left|e_{-1}\right\rangle$ transition.

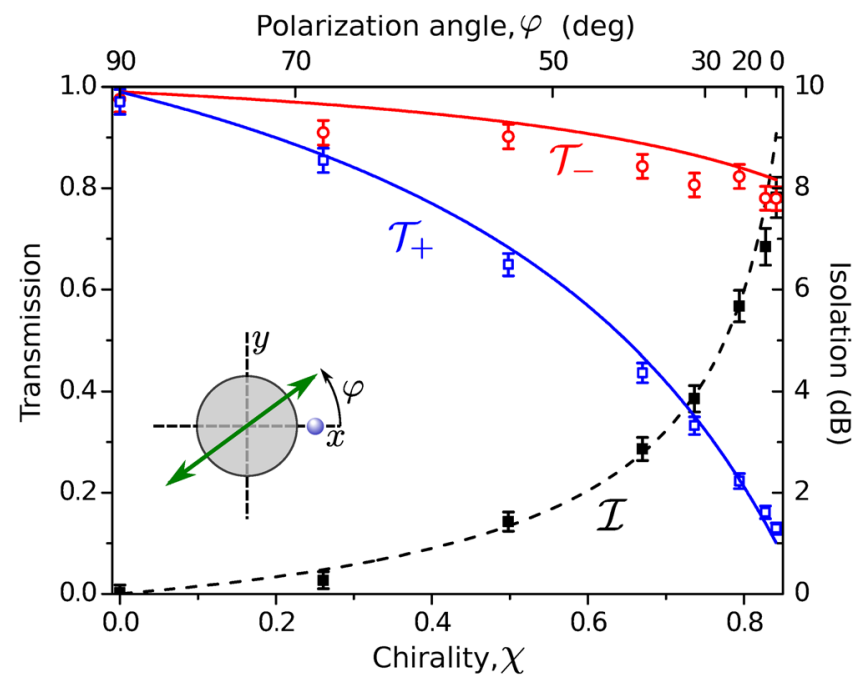

FIG. 3. Nonreciprocal transmission of chiral photons that interact with an ensemble of spin-polarized atoms. Transmissions $\mathcal{T}_{-}$(red) and $\mathcal{T}_{+}$(blue) and isolation $\mathcal{I}$ (black dashed line) as a function of the chirality $\chi$, calculated at the position of the atoms. The lines are the result of a numerical calculation with $\langle N\rangle \approx 27$. The error bars indicate the $1 \sigma$ statistical error based on counting statistics. Inset: Cross section of the optical nanofiber (gray disk) including the trapped atoms (blue sphere) and the main polarization axis of the guided field (green double arrow). The main polarization axis of the guided field and the $x$ axis enclose the angle $\varphi$.

In order to increase the isolation $\mathcal{I}$, more atoms could be loaded into the trap. In the present experiment, the number of atoms is constrained by the limited cesium background pressure in the experiment chamber, and is significantly smaller than what has already been reported in

TABLE I. Experimental results and predictions of the transmissions $\mathcal{T}_{-}$and $\mathcal{T}_{+}$and of the isolation $\mathcal{I}$ for both the ensemble experiment and the resonator experiment. The chirality of the electric field calculated at the position of the atoms is also given. For the ensemble experiment, the predictions are calculated by assuming a mean number of atoms of $\langle N\rangle \approx 27$. For comparison, we also show the predicted values for the case without magnetic offset field.

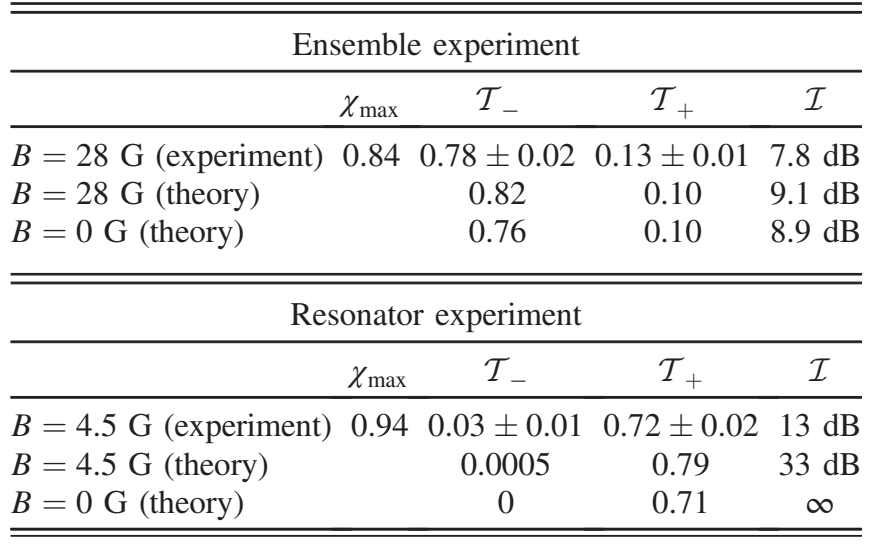


Refs. [28,36]. Remarkably, with only $\langle N\rangle \approx 90$, one would already obtain an isolation of $\mathcal{I}=30 \mathrm{~dB}$, while the transmission $\mathcal{T}_{-}$would remain as high as 0.47 .

Another straightforward approach seems to be to enhance the atom-light interaction strength. Ultimately, one approaches the situation discussed in the proposal by Shen et al. [25]. In this case, the guided light is fully circularly polarized at the position of the emitter and the emitter couples perfectly to the waveguide, i.e., $\beta_{+}^{(+)}=\beta_{-}^{(-)}=1$. In contrast to the argumentation of Ref. [25], however, in such a situation, the waveguide exhibits no nonreciprocal behavior: On a cycling transition, as considered here, the atom emits all the light back into the fiber and into the same direction. Contrarily to our scheme, scattering by the emitters thus does not lead to loss and no nonreciprocal transmission occurs. As a consequence, enhancing the atom-nanofiber coupling cannot, beyond a certain limit, enhance the nonreciprocal behavior.

\section{B. A single resonator-enhanced atom}

In order to realize an optical diode, a single atom can be sufficient in the situation where either $\beta_{+}$or $\beta_{-}$equals 0.5 [27]. In this case, the excitation light field in the optical fiber and the light field that is coherently forward scattered by the atom interfere fully destructively, yielding $\mathcal{T}_{+}=0$ or $\mathcal{T}_{-}=0$, respectively. In order to reach the critical coupling condition $\beta_{-}=0.5$, we need to enhance the atom-waveguide coupling. To this end, we couple a WGM bottle microresonator [20] to the optical nanofiber; see Fig. 2(c). The resonator simultaneously provides strong atom-light coupling and strong transverse confinement of light. In the evanescent field, the chirality reaches $\chi_{\max }=$ 0.94 [20]. In our experiment, a single ${ }^{85} \mathrm{Rb}$ atom is coupled to the resonator mode and prepared in the outermost $m_{F}=$ 3 Zeeman substate of the $F=3$ hyperfine ground state. The states $\left|e_{+1}\right\rangle$ and $\left|e_{-1}\right\rangle$ then correspond to the excited states $\left|F^{\prime}=4, m_{F}=4\right\rangle$ and $\left|F^{\prime}=4, m_{F}=2\right\rangle$, respectively. We apply a magnetic field $B=4.5 \mathrm{G}$ along the resonator axis, and tune the resonator and the nanofiberguided light into resonance with the $|g\rangle \rightarrow\left|e_{+1}\right\rangle$ transition. As in the ensemble experiment, the transition $|g\rangle \rightarrow\left|e_{-1}\right\rangle$ is much weaker than the $|g\rangle \rightarrow\left|e_{+1}\right\rangle$ transition. Thus, the coupling strengths $g_{\circlearrowright, \circlearrowleft}$ between the atom and the two counterrotating resonator modes are determined by their coupling to the $|g\rangle \rightarrow\left|e_{+1}\right\rangle$ transition: They strongly depend on the polarization of the modes and, thus, on their propagation direction. Their ratio is $g_{\circlearrowright} / g_{\circlearrowleft}=5.8$; see Appendix C.

The combined atom-resonator system plays the role of an effective two-level atom: It possesses two states, namely, the clockwise $(i=\circlearrowright)$ and counterclockwise $(i=\circlearrowleft)$ modes of the optical resonator, which are dressed by the atom. More precisely, a nanofiber-guided light field that propagates in the $(+z)[(-z)]$ direction couples solely to the clockwise [counterclockwise] resonator mode with a coupling rate $\kappa$. This means that $\kappa_{-}^{(\circlearrowleft)}=\kappa_{+}^{(\circlearrowright)}=\kappa$ and $\kappa_{+}^{(\circlearrowleft)}=\kappa_{-}^{(\circlearrowright)}=0$. Because of the presence of the atom, the loss rate of the two modes is changed and now reads [41]

$$
\gamma^{(i)}=\gamma_{\mathrm{int}}+g_{i}{ }^{2} / \gamma_{\mathrm{Rb}},
$$

where $2 \gamma_{\mathrm{Rb}}=2 \pi \times 6 \mathrm{MHz}$ is the spontaneous decay rate of the $\left|e_{ \pm 1}\right\rangle$ states, $\gamma_{\text {int }}=2 \pi \times 5 \mathrm{MHz}$ is the intrinsic resonator field decay rate, and $i \in\{\circlearrowright, \circlearrowleft\}$. When the light field is resonant with both the bare resonator and the atom, the transmission through the nanofiber is then given as before as

$$
t_{+}=1-2 \beta_{+}^{(\circlearrowright)} \quad \text { and } \quad t_{-}=1-2 \beta_{-}^{(\circlearrowleft)} .
$$

Because of the different atom-resonator coupling strengths $g_{\circlearrowleft}$ and $g_{\circlearrowright}$, the effective loss rates $\gamma_{\circlearrowleft}$ and $\gamma_{\circlearrowright}$ of the two resonator modes are different. As a consequence, $\beta_{+}^{(\circlearrowright)} \neq$ $\beta_{-}^{(\circlearrowleft)}$ and a strongly nonreciprocal transmission occurs. Remarkably, one obtains perfect isolation, i.e., $t_{-}$or $t_{+}$is zero, for $\kappa=\gamma^{(\circlearrowleft)}$ or $\kappa=\gamma^{(\circlearrowright)}$, which correspond to the case $\beta_{-}^{(\circlearrowleft)}=0.5$ or $\beta_{+}^{(\circlearrowright)}=0.5$, respectively; see Eq. (3). For very large coupling strengths $\kappa$, i.e., for $\beta_{+}^{(\circlearrowright)} \approx \beta_{-}^{(\circlearrowleft)} \approx 1$, the nonreciprocal behavior vanishes and both the transmissions in the $(+z)$ and $(-z)$ direction approach unity.

Figure 4 shows the power transmissions $\mathcal{T}_{+}=\left|t_{+}\right|^{2}$ and $\mathcal{T}_{-}=\left|t_{-}\right|^{2}$ as well as the isolation $\mathcal{I}$ as a function of the nanofiber-resonator coupling strength $\kappa$ for our experimental parameters, where $g_{\circlearrowright}=2 \pi \times 17 \mathrm{MHz}$ and $g_{\circlearrowleft}=$ $2 \pi \times 2.9 \mathrm{MHz}$; see Appendix C. Here, we go beyond the effective two-level atom picture that was used to derive Eq. (3) above: We take into account the full Zeeman

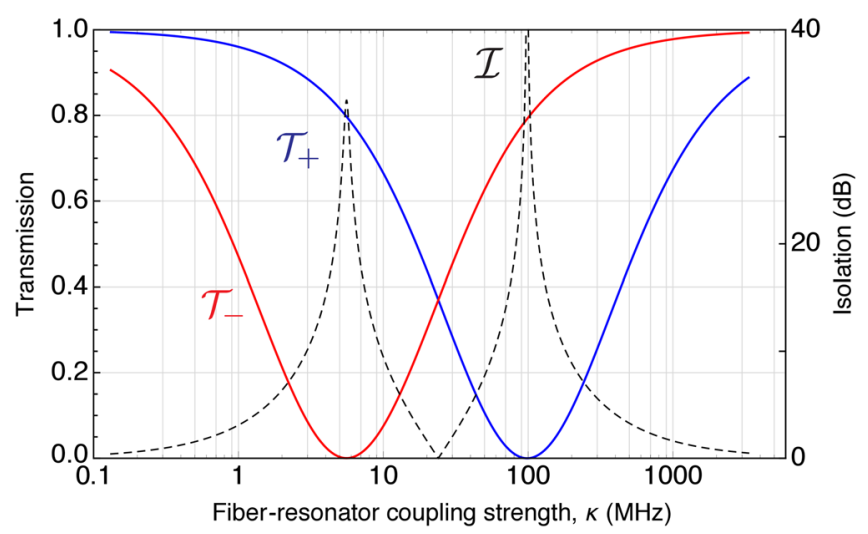

FIG. 4. Calculated nonreciprocal transmission of chiral photons that interact with a single spin-polarized resonator-enhanced atom. The transmission through the nanofiber in $(+z)\left(\mathcal{T}_{+}\right.$, blue solid line) and $(-z)$ directions $\left(\mathcal{T}_{-}\right.$, red solid line) and the isolation ( $\mathcal{I}$, black dashed line) are plotted as a function of the fiber-resonator coupling strength $\kappa$. A magnetic field of $B=$ $4.5 \mathrm{G}$ is assumed to be applied along the resonator axis. 
substructure of the atom and the effect of the applied magnetic field. In accordance with the prediction of the two-level model, we obtain two points of high isolation. However, in contrast to the simple model, the minimum value of $\mathcal{T}_{-}$is finite. This originates from the Zeeman shift of the $|g\rangle \rightarrow\left|e_{-1}\right\rangle$ transition, which is thus detuned from the frequency of the probe light. The resulting dispersive interaction leads to an additional phase shift that inhibits fully destructive interference.

In the experiment, we adjust the resonator-nanofiber coupling strength by changing the fiber-resonator distance. We choose the point $\kappa=\gamma_{\text {int }}=2 \pi \times 5 \mathrm{MHz}$, which corresponds to critical coupling to the empty resonator and is close to the optimal value of $\kappa=\gamma^{(\circlearrowleft)} \approx 2 \pi \times 5.5 \mathrm{MHz}$ obtained from the full model. The power of the probe light field is $3 \mathrm{pW}$, corresponding to $\approx 0.2$ photons per resonator lifetime. We measure a transmission through the nanofiber of $\mathcal{T}_{+}=0.72 \pm 0.02$ in the $(+z)$ direction and $\mathcal{T}_{-}=$ $0.03 \pm 0.01$ in the $(-z)$ direction. This corresponds to an isolation of $\mathcal{I}=13 \mathrm{~dB}$. The maximum isolation predicted by theory is $33 \mathrm{~dB}$; see Table I. The deviation of the experimental value from this number stems from two effects. First, the fiber-resonator coupling strength is slightly lower than the value for optimum isolation. Second, and more importantly, both the laser-resonator detuning and the fiber-resonator coupling strength exhibit technical fluctuations.

An important aspect of our system is that the nonreciprocal transmission properties depend on the internal state of the atom. In order to show this, we measure the transmission through the nanofiber in the $(-z)$ direction as a function of time; see Fig. 5(a). In this case, the light that enters the resonator is nearly fully $\sigma^{-}$polarized and can drive $\Delta m_{F}=-1$ transitions. Although the coupling strength to the $|g\rangle \rightarrow\left|e_{-1}\right\rangle$ transition is very weak, it can still be driven and the atom will eventually be pumped to the state $\left|F=3, m_{F}=-3\right\rangle$. For this atomic state, the directional behavior of the system is reversed because the atom is strongly coupled to the counterclockwise resonator mode. We now observe a high transmission through the waveguide in the $(-z)$ direction. The strength and direction of the optical isolation can thus be controlled via the spin of the atom.

In contrast to the ensemble-based scheme, the resonatorbased approach relies on a single atom only. We confirm this by measuring the second-order correlation function of the light transmitted through the nanofiber in the $(+z)$ direction. Indeed, we expect a highly nonlinear response already when only two photons arrive simultaneously [41]. Figure 5(b) shows the measured correlation function (blue line), which exhibits a clear signal of photon antibunching. Using our experimental parameters, we model this behavior (red line), which perfectly agrees with our measured data; see Appendix C. We note that in contrast to typical resonance fluorescence measurements, in our situation,
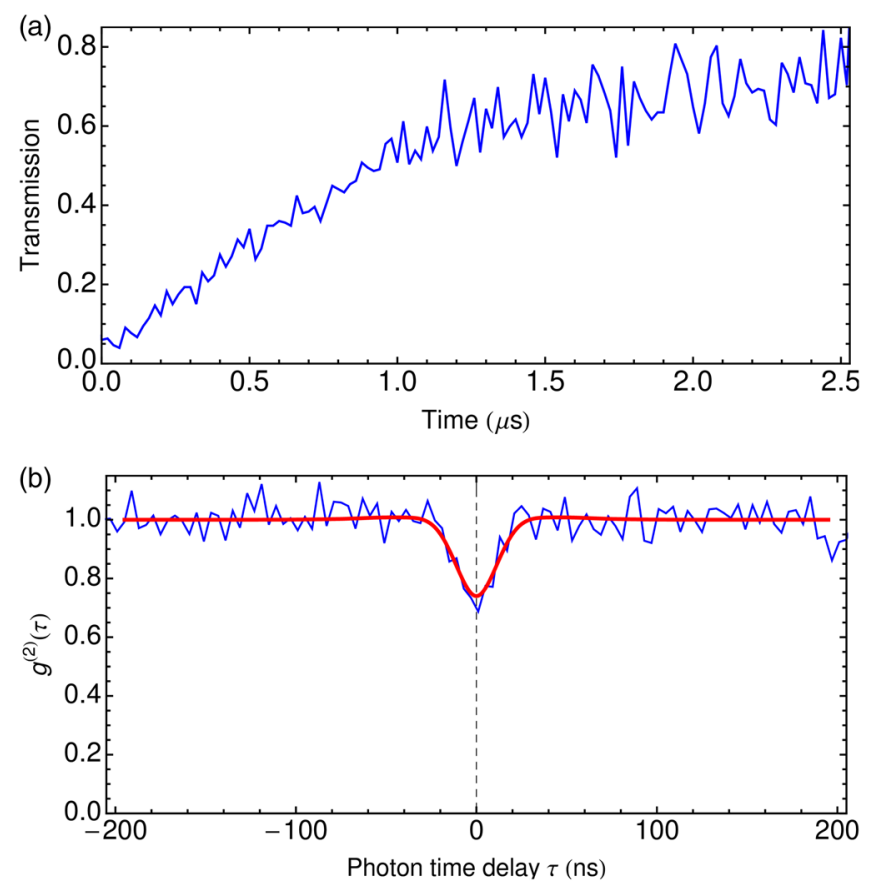

FIG. 5. Control of the nonreciprocal transmission using a single resonator-enhanced atom. (a) Measured transmission $\mathcal{T}_{-}$as function of time. Because of optical pumping into the Zeeman ground state $\left|F=3, m_{F}=-3\right\rangle$, the system changes its transmission properties and, after about $2 \mu \mathrm{s}$, the directionality of the optical diode reverses. (b) Second-order correlation function $g^{(2)}$ of the light transmitted through the nanofiber in the $(+z)$ direction. The thin blue (thick red) line corresponds to the measured data (theoretical prediction). For zero time delay one observes photon antibunching.

independent of the setting of $\kappa$, the $g^{(2)}$ function never reaches zero since the transmitted field always contains a contribution from the incident coherent light field. Our data thus confirm that a single atom couples to the resonator.

The observed antibunching also demonstrates that the atom-resonator system constitutes a nonlinear isolator at the single-photon level, where the transmission through the fiber strongly depends on the number of photons in the waveguide. This nonlinear behavior depends on the parameters of the atom-resonator system and can, in principle, be adjusted to achieve minimal nonlinearity for classical diode operation as well as for strong nonlinearity as required for photon number-dependent operation.

\section{Discussion}

For the practical implementation of the demonstrated isolator concept, certain requirements have to be fulfilled. For a continuous operation, the atoms have to be coupled to the fiber-guided light over long time periods. This requires a trapping scheme for atoms close to the resonator as well as an optimization of the trapping and loading scheme for both setups to significantly increase the current duty cycles of around $10^{-6}$ (resonator case) and $10^{-4}$ (ensemble case). 
Furthermore, as can be seen from Fig. 5, the performance of the demonstrated diodes depends strongly on the internal state of the atoms. Therefore, for practical applications it is important that the atomic state does not change over time, e.g., due to optical depumping by fiber-guided light. In this context, there are fundamental differences between the two implementations: In the ensemble experiment, it is the light that propagates in the forward direction that alters the initial atomic state in such a way that the forward transmission is lowered and the direction of the diode eventually reverses. For the resonator-enhanced atom, it is the backwardpropagating light that leads to this effect. In this case, the optical pumping effects of the forward-propagating light field are opposite, meaning that it permanently pumps the atoms back into the state desired for the diode operation. In principle, this even enables the continuous operation of the diode as long as the power of the backward-propagating light is small enough. In both schemes, unwanted optical pumping can always be counteracted by using an external or an additional fiber-guided light field that permanently pumps the emitters towards the desired internal state. Choosing this light to be resonant to a different optical transition than the one used for the operation of the isolator would then enable continuous operation of the diode. Another crucial parameter is the upper power limit of the guided light field. It is set by the saturation intensity of the atoms and the atom-light coupling strength. For the demonstrated experimental realizations, this yields an upper power limit on the order of $10 \mathrm{pW}$. In order to achieve operation at higher power levels for a given isolation and forward transmission, the emitterwaveguide coupling strength has to be decreased while simultaneously increasing the number of emitters.

For future applications, the resonator-enhanced atom case is ideally suited for low optical power, where it can reach, in principle, perfect isolation. In particular, since the diode is controlled by a single atom only, this also enables quantum applications in which the diode is prepared in a superposition of, e.g., transmitting in the $(+z)$ and in the $(-z)$ direction. From a practical point of view, the ensemble-based system requires less experimental overhead because the emitters are directly coupled to the waveguide. Moreover, it is less sensitive to fluctuations in the emitter-waveguide coupling strength, which has to be precisely adjusted in the resonator-enhanced scheme.

The demonstrated isolator concept is compatible with a large variety of emitters and optical waveguide structures. By replacing the atoms with solid-state emitters like quantum dots, dopant ions, or defect centers and by exchanging the optical nanofibers used here with integrated photonic waveguides, one could realize an optical chip-based design and increase the number of coupled emitters significantly. Another possible avenue for implementing the scheme in practical devices is the use of plasmonic systems. In plasmonics, the realization of polarization-dependent coupling is a matter of current research. If a suitable active or passive plasmonic structure could be found, this would be a major breakthrough for the practical implementation of our scheme: Apart from making optical pumping dispensable, plasmonics does not suffer from saturation effects and is compatible with lithographic fabrication technologies and room temperature operation.

\section{CONCLUSION}

In summary, we perform proof-of-concept experiments demonstrating nanophotonic optical isolators that operate at the single-photon level and with low loss. We take advantage of the occurrence of a transverse spin in the evanescent field of guided light and employ its chiral coupling to spin-polarized quantum emitters. The direction of the optical diode is controlled by the spin of the emitters. We believe that our results lay the foundations for a new class of nanophotonic devices based on chiral light-matter interaction which complement the existing components for conventional integrated optical signal processing.

Our concept is compatible with ultralow light levels down to single photons, a prerequisite for quantum applications. This then opens up novel ways of quantum information processing based on chiral light-matter interaction $[21,42,43]$ and should, e.g., allow one to implement quantum protocols in which the nonreciprocity is used to generate entanglement $[44,45]$. Instead of operating the nonreciprocal waveguide in the dissipative regime, it would also be possible to realize dispersive nonreciprocal elements [27] and thus to, e.g., generate nonclassical states of light. The quantum emitter can also be prepared in a superposition state which further widens the range of potential applications as it enables the realization of optical quantum elements such as quantum circulators.

\section{ACKNOWLEDGMENTS}

Financial support by the Austrian Science Fund (FWF, SFB NextLite Project No. F 4908-N23, SFB FoQuS Project No. F 4017, and DK CoQuS Project No. W 1210-N16) and the European Commission (IP SIQS, No. 600645) is gratefully acknowledged. C.S. and J. V. acknowledge support by the European Commission (Marie Curie IEF Grants No. 328545 and No. 300392, respectively). C. J. acknowledges support from the German National Academic Foundation.

\section{APPENDIX A: OPTICAL NANOFIBER}

The silica optical nanofiber is realized as the waist of a tapered optical fiber. This enables close-to-unity coupling efficiency of light from the unprocessed fiber to the nanofiber waist and vice versa. The nanofiber has a nominal radius of $a=250 \mathrm{~nm}$, which is small enough so that the fiber only guides the fundamental $\mathrm{HE}_{11}$ mode for all wavelengths involved in the experiments. 


\section{APPENDIX B: ENSEMBLE EXPERIMENT}

The atoms are located in a nanofiber-based two-color optical dipole trap [28]. Two diametric linear arrays of trapping sites are created by sending a 1064-nmwavelength red-detuned standing wave, with $0.77 \mathrm{~mW}$ per beam, and a blue-detuned running wave with a 783-nmwavelength and a power of $8.5 \mathrm{~mW}$ through the nanofiber. The period of the array is about $0.5 \mu \mathrm{m}$, the sites are located $230 \mathrm{~nm}$ from the nanofiber surface, and they contain at most one atom [28].

In this work, for technical reasons, we only use the atoms in one of the two linear arrays, namely, in the one located at $x>a$; see Fig. 2(b). Initially, the atoms are optically pumped using a nanofiber-guided light field. As outlined in Ref. [38], the atoms on one side of the fiber are pumped into the state $\left|F=4, m_{F}=+4\right\rangle$ and the atoms on the other side are pumped into $\left|F=4, m_{F}=-4\right\rangle$. The magnetic offset field of $28 \mathrm{G}$ that stabilizes the atomic spin states lifts the degeneracy of the cycling transitions, $\left|F=4, m_{F}=+4\right\rangle \rightarrow$ $\left|F^{\prime}=5, m_{F^{\prime}}=+5\right\rangle$, and $\left|F=4, m_{F}=-4\right\rangle \rightarrow\left|F^{\prime}=5, m_{F^{\prime}}=-5\right\rangle$. We tune the probe light field into resonance with the $\left|F=4, m_{F}=+4\right\rangle \rightarrow\left|F^{\prime}=5, m_{F^{\prime}}=+5\right\rangle \quad$ transition. It then effectively interacts only with atoms on one side of the nanofiber. We note that the diode would also operate if the probe light coupled to atoms on both sides of the fiber, i.e., if the two cycling transitions were still degenerate: Given that the probe field has the same polarization properties as the field that optically pumps the atoms, the ensembles on both sides of the fiber would individually lead to optical isolation in the same direction and their contributions would add up.

The theoretical predictions plotted in Fig. 3 are the result of numerical calculations. The latter are based on the model developed in Ref. [37]. The transmissions $\mathcal{T}_{ \pm}(N=1)$ are calculated for a single atom prepared in the $\left|F=4, m_{F}=4\right\rangle$ state, located $230 \mathrm{~nm}$ from the surface of a $250 \mathrm{~nm}$-radius nanofiber. The model takes into account the value of the offset magnetic field and the orientation of the main polarization of the guided light field. The transmissions corresponding to a mean number of trapped atoms of $\langle N\rangle$ are given by

$$
\mathcal{T}_{ \pm}(\langle N\rangle)=\sum_{N} p(N,\langle N\rangle) \mathcal{T}_{ \pm}(N=1)^{N},
$$

where $p(N,\langle N\rangle)$ is the Poisson distribution of average $\langle N\rangle$. The mean number of atoms $\langle N\rangle \approx 27$ used in Fig. 3 is the result of a fit to the experimental data.

\section{APPENDIX C: RESONATOR EXPERIMENT}

The bottle microresonator is a highly prolate shaped WGM resonator with a diameter of $36 \mu \mathrm{m}$ fabricated from a standard optical fiber. Its coupling $\kappa$ to the optical nanofiber is tuned by changing the distance between the nanofiber and the resonator surface.
In the experiment, an atomic fountain delivers a cloud of laser-cooled ${ }^{85} \mathrm{Rb}$ atoms to the resonator. In order to detect the presence of a single atom in the resonator mode in real time, we critically couple the optical nanofiber and the resonator. A single-photon-counting module records the transmission through the nanofiber of a probe light field that is resonant with the empty resonator mode [20]. When an atom enters the resonator mode, the transmission increases by two orders of magnitude and the interaction with the resonator light field optically pumps the atom into the $\left|F=3, m_{F}=3\right\rangle$ hyperfine ground state. Using a field programmable gate array-based real-time detection and control system, we react to the increasing count rate within approximately $150 \mathrm{~ns}$. Subsequently, we execute our measurement sequence during which we send a light pulse through the nanofiber for a predetermined time interval ranging from $100 \mathrm{~ns}$ to a few microseconds along the $(+z)$ or $(-z)$ direction. A final 1- $\mu$ s-probing interval ensures that the atom is still coupled to the resonator mode at the end of the measurement sequence.

\section{Atom-resonator interaction}

In order to obtain the theoretical predictions for the atomresonator system shown in Fig. 4, we start with the JaynesCummings Hamiltonian that describes the interaction of a two-level atom with an optical mode. We extend this model to include the two counterpropagating resonator modes as well as their chiral character and the full Zeeman substructure of the atom. The only free parameter is the average coupling strength $g_{\circlearrowright}$ between a single atom and the resonator mode that propagates in the clockwise direction. From a measurement of the vacuum-Rabi splitting [20], we obtain $g_{\circlearrowright}=2 \pi \times 17 \mathrm{MHz}$. The ratio between $g_{\circlearrowright}$ and $g_{\circlearrowleft}$ can be approximated by the coupling strength of the two counterpropagating resonator modes to the $|g\rangle \rightarrow$ $\left|e_{+1}\right\rangle$ transition, which for $\chi=0.94$ yields $g_{\circlearrowright} / g_{\circlearrowleft}=5.8$.

\section{Atom-resonator interaction beyond the single-photon limit}

In order to simulate the behavior of the atom-resonator system for the case with more than one photon present, we perform a full numerical calculation of our system by solving the master equations of the pumped atom-resonator system using the approximation of a two-level atom interacting with a single resonator mode [20]. In this case, the master equation is given by

$$
\begin{aligned}
\frac{d \rho(t)}{d t}= & -\frac{i}{\hbar}[H, \rho]+\left(\kappa_{f}+\kappa_{i}\right)\left(2 b \rho b^{\dagger}-b^{\dagger} b \rho-\rho b^{\dagger} b\right) \\
& +\gamma\left(2 \sigma^{-} \rho \sigma^{+}-\sigma^{+} \sigma^{-} \rho-\rho \sigma^{+} \sigma^{-}\right),
\end{aligned}
$$

where

$$
\begin{aligned}
H / \hbar= & \Delta_{r l} b^{\dagger} b+\Delta_{a l} \sigma^{+} \sigma^{-}+g\left(b^{\dagger} \sigma^{-}+b \sigma^{+}\right) \\
& +\sqrt{2 \kappa_{f}}\left\langle a_{\text {in }}\right\rangle\left(b^{\dagger}+b\right)
\end{aligned}
$$


is the atom-resonator Hamiltonian in the rotating wave approximation. Here, $\Delta_{r l}\left(\Delta_{a l}\right)$ is the resonator-light (atomlight) detuning, $b\left(b^{\dagger}\right)$ is the annihilation (creation) operator of a resonator photon, $\sigma^{+}\left(\sigma^{-}\right)$is the atomic excitation (deexcitation) operator, $\rho$ is the atom-resonator density matrix, and $\left\langle a_{\text {in }}\right\rangle$ is the amplitude of the incident fiberguided light. The final state of the light field after the interaction with the resonator is given by the interference of the field in the fiber with the field that is coupled out of the resonator:

$$
a_{\text {out }}=a_{\text {in }}-\sqrt{2 \kappa_{f}} b .
$$

The input light field corresponds to a coherent state that allows us to replace the input operator $a_{\text {in }}$ by its expectation value [46]. Using the measured distribution of coupling strengths, we calculate the expectation values for the output fields, which allows us to predict, e.g., the second-order correlation function of the fiber field $a_{\text {out }}$.

[1] See Focus: Silicon Photonics, Nat. Photonics 4, 491 (2010).

[2] J. L. O’Brien, Science 318, 1567 (2007).

[3] H. J. Kimble, Nature (London) 453, 1023 (2008).

[4] T. R. Zaman, X. Guo, and R. J. Ram, J. Lightwave Technol. 26, 291 (2008).

[5] K. Gallo, G. Assanto, K. R. Parameswaran, and M. M. Fejer, Appl. Phys. Lett. 79, 314 (2001).

[6] L. Fan, J. Wang, L. T. Varghese, H. Shen, B. Niu, Y. Xuan, A. M. Weiner, and M. Qi, Science 335, 447 (2012).

[7] B. Peng, S. K. Ozdemir, F. Lei, F. Monifi, M. Gianfreda, G. L. Long, S. Fan, F. Nori, C. M. Bender, and L. Yang, Nat. Phys. 10, 394 (2014).

[8] H. Lira, Z. Yu, S. Fan, and M. Lipson, Phys. Rev. Lett. 109, 033901 (2012).

[9] L. D. Tzuang, K. Fang, P. Nussenzveig, S. Fan, and M. Lipson, Nat. Photonics 8, 701 (2014).

[10] N. A. Estep, D. L. Sounas, J. Soric, and A. Alu, Nat. Phys. 10, 923 (2014).

[11] J. Kim, M. C. Kuzyk, K. Han, H. Wang, and G. Bahl, Nat. Phys. 11, 275 (2015).

[12] D. K. Linkhart, Microwave Circulator Design, 2nd ed. (Artech House, Norwood, 2014).

[13] K. Y. Bliokh, A. Aiello, and M. A. Alonso, The Angular Momentum of Light, edited by D. L. Andrews and M. Babiker (Cambridge University Press, Cambridge, England, 2012).

[14] A. B. Fuller, Ferrites at Microwave Frequencies, Electromagnetic Waves Series Vol. 23 (The Institution of Engineering and Technology, London, 1986).

[15] R. J. Potton, Rep. Prog. Phys. 67, 717 (2004).

[16] Y. Shoji, T. Mizumoto, H. Yokoi, I.-W. Hsieh, and R. M. Osgood, Appl. Phys. Lett. 92, 071117 (2008).

[17] M.-C. Tien, T. Mizumoto, P. Pintus, H. Kromer, and J. E. Bowers, Opt. Express 19, 11740 (2011).

[18] L. Bi, J. Hu, P. Jiang, D. H. Kim, G. F. Dionne, L. C. Kimerling, and C. A. Ross, Nat. Photonics 5, 758 (2011).

[19] Y. Shoji and T. Mizumoto, Sci. Tech. Adv. Mater. 15, 014602 (2014).
[20] C. Junge, D. O'Shea, J. Volz, and A. Rauschenbeutel, Phys. Rev. Lett. 110, 213604 (2013).

[21] I. Shomroni, S. Rosenblum, Y. Lovsky, O. Bechler, G. Guendelman, and B. Dayan, Science 345, 903 (2014).

[22] I. J. Luxmoore, N. A. Wasley, A. J. Ramsay, A. C. T. Thijssen, R. Oulton, M. Hugues, A. M. Fox, and M. S. Skolnick, Appl. Phys. Lett. 103, 241102 (2013).

[23] R. Mitsch, C. Sayrin, B. Albrecht, P. Schneeweiss, and A. Rauschenbeutel, Nat. Commun. 5, 5713 (2014).

[24] I. Sölllner, S. Mahmoodian, S. L. Hansen, L. Midolo, A. Javadi, G. Kiršanskè, T. Pregnolato, H. El-Ella, E. H. Lee, J. D. Song, S. Stobbe, and P. Lodahl, Nat. Nanotechnol. 10, 775 (2015).

[25] Y. Shen, M. Bradford, and J.-T. Shen, Phys. Rev. Lett. 107, 173902 (2011).

[26] E. J. Lenferink, G. Wei, and N. P. Stern, Opt. Express 22, 16099 (2014).

[27] K. Xia, G. Lu, G. Lin, Y. Cheng, Y. Niu, S. Gong, and J. Twamley, Phys. Rev. A 90, 043802 (2014).

[28] E. Vetsch, D. Reitz, G. Sagué, R. Schmidt, S. T. Dawkins, and A. Rauschenbeutel, Phys. Rev. Lett. 104, 203603 (2010).

[29] K. Y. Bliokh and F. Nori, Phys. Rev. A 85, 061801 (2012).

[30] M. Neugebauer, T. Bauer, P. Banzer, and G. Leuchs, Nano Lett. 14, 2546 (2014).

[31] J. Petersen, J. Volz, and A. Rauschenbeutel, Science 346, 67 (2014).

[32] B. le Feber, N. Rotenberg, and L. Kuipers, Nat. Commun. 6, 6695 (2015).

[33] F. J. Rodriguez-Fortuno, I. Barber-Sanz, D. Puerto, A. Griol, and A. Martinez, ACS Photonics 1, 762 (2014).

[34] D. Jalas, A. Petrov, M. Eich, W. Freude, S. Fan, Z. Yu, R. Baets, M. Popovic, A. Melloni, J. D. Joannopoulos, M. Vanwolleghem, C. R. Doerr, and H. Renner, Nat. Photonics 7, 579 (2013).

[35] D. Steck, Cesium D Line Data, http://steck.us/alkalidata/ cesiumnumbers.pdf.

[36] E. Vetsch, S. T. Dawkins, R. Mitsch, D. Reitz, P. Schneeweiss, and A. Rauschenbeutel, IEEE J. Sel. Top. Quantum Electron. 18, 1763 (2012).

[37] Fam Le Kien and A. Rauschenbeutel, Phys. Rev. A 90, 023805 (2014).

[38] R. Mitsch, C. Sayrin, B. Albrecht, P. Schneeweiss, and A. Rauschenbeutel, Phys. Rev. A 89, 063829 (2014).

[39] D. Reitz, C. Sayrin, R. Mitsch, P. Schneeweiss, and A. Rauschenbeutel, Phys. Rev. Lett. 110, 243603 (2013).

[40] Fam Le Kien, S. Dutta Gupta, V. I. Balykin, and K. Hakuta, Phys. Rev. A 72, 032509 (2005).

[41] J. Volz, M. Scheucher, C. Junge, and A. Rauschenbeutel, Nat. Photonics 8, 965 (2014).

[42] S. Rosenblum and B. Dayan, arXiv:1412.0604.

[43] T. C. Ralph, I. Söllner, S. Mahmoodian, A. G. White, and P. Lodahl, Phys. Rev. Lett. 114, 173603 (2015).

[44] K. Stannigel, P. Rabl, and P.Zoller, New J. Phys. 14, 063014 (2012).

[45] T. Ramos, H. Pichler, A. J. Daley, and P. Zoller, Phys. Rev. Lett. 113, 237203 (2014).

[46] C. W. Gardiner and M. J. Collett, Phys. Rev. A 31, 3761 (1985). 